International Journal of Modern Physics D, Vol. 9, No. 6 (2000) 633-647

(E-print arXiv:gr-qc/0004054, v2 with only a few corrections).

\title{
A Generalized Equivalence Principle
}

\author{
HOLGER LYRE \\ Institut für Philosophie, Ruhr-Universität Bochum, D-44780 Bochum, Germany, \\ e-mail: holger.lyre@ruhr-uni-bochum.de
}

April 2000

\begin{abstract}
Gauge field theories may quite generally be defined as describing the coupling of a matter-field to an interaction-field, and they are suitably represented in the mathematical framework of fiber bundles. Their underlying principle is the socalled gauge principle, which is based on the idea of deriving the coupling structure of the fields by satisfying a postulate of local gauge covariance. The gauge principle is generally considered to be sufficient to define the full structure of gauge-field theories. This paper contains a critique of this usual point of view: firstly, by emphazising an intrinsic gauge theoretic conventionalism which crucially restricts the conceptual role of the gauge principle and, secondly, by introducing a new generalized equivalence principle - the identity of inertial and field charge (as generalizations of inertial and gravitational mass) - in order to give a conceptual justification for combining the equations of motion of the matter-fields and the field equations of the interactionfields.
\end{abstract}

\section{Content}

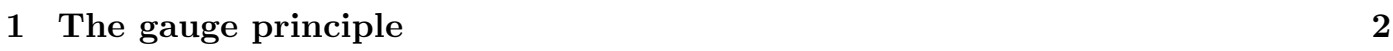

\begin{tabular}{|lll|}
\hline 2 & The intrinsic gauge theoretic conventionalism & 3
\end{tabular}

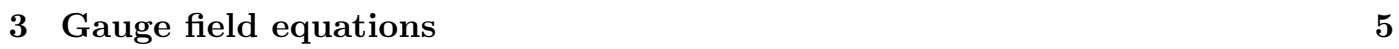

\begin{tabular}{|lll}
\hline 4 & The lesson from general relativity & 6
\end{tabular}

4.1 The gauge theoretic analogy . . . . . . . . . . . . . . . . . . . . . 6

4.2 The equivalence principle . . . . . . . . . . . . . . . . . . . . . . 8

\begin{tabular}{|lll}
5 & A generalized equivalence principle & 9
\end{tabular}

5.1 The "missing link" . . . . . . . . . . . . . . . . . . . . . 9 9

$5.2 \quad$ Yang-Mills theories, charges and couplings . . . . . . . . . . . . . . . . . . . . 10

5.3 Experimental conclusions . . . . . . . . . . . . . . . . . . . . 12

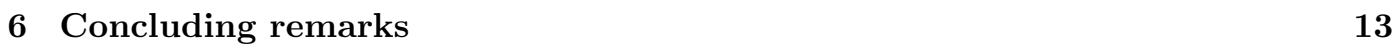




\section{The gauge principle}

At least three of the four known fundamental interactions are representable as gauge-field theories (for short, 'gauge theories'). Thus, from the conceptual point of view it is of particular interest to understand the role, effect and explanatory power of the possible underlying principles of this kind of theories. Now, it is quite customary to consider the so-called gauge principle (GP) as the one and only and, hence, sufficient principle to almost dictate the full structure of gauge-field theories by merely satisfying a postulate of local gauge covariance. However, as we shall see, this turns out too strong a statement.

It is the purpose of the first three sections of this paper to present a detailed analysis of the GP's true conceptual role. To begin with, recall the DiRAC-MAXWELL theory as the paradigm gauge theory. Note that it is the basic idea of gauge-field theories to represent the combination of two field theories: a matter-field and an interaction-field or, in our example, the DIRAC and the Maxwell fields. In the spirit of the GP we must start with a free matter-field theory, represented by the DiRAC Lagrangian

$$
\mathcal{L}_{D}=\bar{\psi}(x)\left(i \gamma^{\mu} \partial_{\mu}-m\right) \psi(x),
$$

where each spinor component $\psi^{i}$ is a $U(1)$ representation. This Lagrangian is clearly invariant under global $U(1)$ gauge transformations $\psi^{\prime}(x)=e^{i q^{(i)}} \Lambda(x)$ (the factor $q^{(i)}$ and its notation will become clear below). For the construction of a $U(1)$ gauge theory we demand covariancef of the free matter-field theory represented by (1) under local (i.e. spacetime-dependent) transformations

$$
\psi(x) \rightarrow \psi^{\prime}(x)=e^{i q^{(i)} \Lambda(x)} \psi(x) \equiv \hat{U}(x) \psi(x) .
$$

This requirement is called the gauge postulate. Inserting (21) in (11) we get an extraneous term $-q^{(i)} \bar{\psi}\left(\gamma^{\mu} \partial_{\mu} \Lambda\right) \psi$, which may be compensated by introducing a vector field

$$
A_{\mu}(x)=\partial_{\mu} \Lambda(x)
$$

with the transformation behaviour

$$
A_{\mu}^{\prime}(x)=A_{\mu}(x)-\partial_{\mu} \Lambda(x) .
$$

In other words, in order to satisfy the postulate of local gauge covariance of the free theory, we have to replace the usual derivative by the so-called covariant derivative

$$
\partial_{\mu} \rightarrow D_{\mu}=\partial_{\mu}+i q^{(i)} A_{\mu} .
$$

Thus, instead of (1), we get a transformed DIRAC Lagrangian

$$
\mathcal{L}_{D}^{\prime}=\bar{\psi}(x)\left(i \gamma^{\mu} D_{\mu}-m\right) \psi(x)
$$

\footnotetext{
${ }^{1}$ Accordingly, quantum electrodynamics is the quantized version of the DIRAC-MAXwELL gauge theory. Note, however, that it is misleading to call MAXWELL's theory, i.e. classical electrodynamics, a gauge theory merely because of its gauge freedom $A_{\mu}^{\prime}(x)=A_{\mu}(x)-\partial_{\mu} \Lambda(x)$; since a true gauge theory couples matter-fields and interaction-fields. I shall strictly use this terminology; cf. LYRE (1999).

${ }^{2}$ Throughout this paper we speak of covariance of theories as opposed to invariance of certain objects of a theory (with respect to some symmetry group).
} 
which is invariant under the combined local gauge transformations (2) and (四).

Mathematically, gauge theories allow for an adaequate representation within the geometrical framework of principal fiber bundles $\mathbb{P}$ and their associated vector bundles $\mathbb{E}$. The bundle approch defines a typical correspondence between the geometrical and the physical terms arising in gauge theories: Gauge potentials are connections of $\mathbb{P}$, local sections of $\mathbb{E}$ represent matterfields. The gauge group is the structure group of the bundle and its generators are the gauge bosons. Local gauge transformations may be regarded as automorphisms of $\mathbb{P}$. Finally, the bundle curvature is to be considered as the interaction-field strength.

\section{The intrinsic gauge theoretic conventionalism}

Usually, the replacement (3) is interpreted as the introduction of a new "interaction-field". Hence, the transformed Lagrangian (6) is considered to describe a theory in which the DIRAC matter-field is coupled to a "field" $A_{\mu}$. Given $A_{\mu}$, it is possible to form the bundle curvature tensor

$$
F_{\mu \nu}=\partial_{\mu} A_{\nu}-\partial_{\nu} A_{\mu}
$$

On the background of the interaction interpretation we are motivated to call $A_{\mu}$ the gauge potential and $F_{\mu \nu}$, its derivative, the gauge-field strength. $]^{3}$ This interpretation is suggested by the following consideration. As mentioned in the previous section, the gauge postulate basically amounts to replacing global by local transformations. Due to NoETHER's first theorem global gauge invariance of some Lagrangian under a $k$-dimensional LIE group is connected with the existence of $k$ conserved currents. For the one-dimensional $U(1)$ we obtain the electric current

$$
\jmath_{\mu}^{(i)}=q^{(i)} \bar{\psi} \gamma_{\mu} \psi
$$

in a notation analogous to $q^{(i)}$. It obeys the continuity equation

$$
\partial_{\mu} \jmath^{(i) \mu}=0 .
$$

Hence, because of the existence of a charged NoETHER current it is indeed suggestive to assume that there exists a true interaction field which couples to this current and which may be represented by its potential $A_{\mu}$. More explicitly,

$$
\mathcal{L}_{\text {int }}^{(i)}=-\jmath_{\mu}^{(i)} A^{\mu}
$$

should be considered as the coupling part of the transformed Lagrangian $\mathcal{L}_{D}^{\prime}$, now written as

$$
\mathcal{L}_{D}^{\prime}=\mathcal{L}_{D}+\mathcal{L}_{\text {int }}^{(i)}
$$

to underline the interaction interpretation.

Note that all of the above derivations arise from the GP "with a little help" from Noether's theorem. From this point of view, the GP appears as a most powerful tool - at first glance

\footnotetext{
${ }^{3}$ In theoretical high energy physics it is common practice to call $A_{\mu}$ a "field", but in order to avoid confusion I shall consistently call $A_{\mu}$ the gauge potential (actually, $F_{\mu \nu}$ describes the real gauge-fields $\vec{E}$ and $\vec{B}$ ).
} 
sufficient to derive the coupling to an interaction-field! But, did it really do this job? Let us reconsider the crucial step of the argument: the local gauge transformation (2) implies an extraneous term basicially consisting of the derivation of the phase function $\Lambda(x)$. However, the "replacement" (3) is of course nothing but a mere renaming. It is nice heuristics to assume $A_{\mu}$ as a real potential which couples to a charged current $j_{\mu}^{(i)}$ - but we are, at this stage, by no means forced to do so. All that has happend is that the Lagrangian was transformed in a way that it looks as if it implied a coupling. What the GP rather leads to is a special sort of an internal coordinate transformation in the local fibers of the bundle space. The coupling may very well be understood as a mathematical artefact arising from a mere bundle coordinate transformation and, hence, representing a pure conventional manoeuvre! I propose to call this an intrinsic gauge theoretic conventionalism. It has no physical significance so far.

To obtain a theory with true physical potentials we need to secure a non-vanishing field strength, which - as a dynamical element and thereby truly changing the physical situation must obey some field equations. Th To underline this point one should recall the bundle structure of gauge theories. Quite generally, the types of bundles arising in gauge theories may be distinguished in a three-fold way:

- type 1: Trivial bundles with flat connections,

- type 2: Trivial bundles with non-flat connections,

- type 3: Non-trivial bundles.

This distinction is a useful one from the point of view of characterizing the possible bundle geometries in gauge theories. Fiber bundles can be considered as generalizations of the direct product of spaces - here: base space and typical fiber. Only locally do they look like a product space. Trivial bundles, however, can even globally be reduced to a direct product. Therefore, type 1 bundles really turn out as superfluous structures. Of course, with mathematical rigour the same is true for type 2 bundles, too. Only type 3 bundles appear to be justified (from this point of view it is not necessary to distinguish between non-trivial bundles with flat or non-flat connections).

\footnotetext{
${ }^{4}$ One might, however, argue that the celebrated AHARONOV-BoHM effect as well as other topological effects in gauge theories point to the physical significance of the potentials alone. This may be conceded, but it does not concern our issue here. We want to understand the principles underlying the full structure of gauge-field theories. The AB effect may then be described as a special result. Moreover, although the effect seems to show a direct influence of the gauge potential on the electron matter-field (in a setting with vanishing field strength), the occurrence of this potential outside the solenoid is only due to the fact that there is a real interaction-field inside the solenoid. Without the existence of this field, of course, there is no AB effect at all. It is only the existence of the internal magnetic field that justifies the interpretation of the vector potential external to the solenoid as a true physical interaction potential.

${ }^{5}$ From the conceptual point of view, however, even type 2 bundles (which actually mainly occur in the experimentally vindicated part of the standard model) may be considered as extremely useful, since gauge theoretic bundles in general allow for a natural distinction between the merely mathematical structures, which live in the base space, and the truly physical structures of gauge-field theories, which live in the fibers; cf. GuTTMANN and LYRE (2000).
} 
Now, a flat connection is defined as a connection with vanishing curvature and therefore physically describing a field-free configuration (i.e. vanishing gauge-field strength). What kind of bundle geometry is then described by the transformed Lagrangian $\mathcal{L}_{D}^{\prime}$ in (16) or (11), respectively? As we saw there is no definite answer to this question, since on the basis of $\mathcal{L}_{D}^{\prime}$ alone we simply cannot decide whether we are dealing with a truly coupled or merely internally transformed Lagrangian. This, again, means that the GP by itself just implies a pure conventional coordinate transformation (in a trivial bundle) - a procedure which does not distinguish between type 1 and type 2 bundles. Thus, in order to give rise to type 2 (or maybe even type 3 ) bundles we have to introduce a real interaction-field dynamics. This shall be analyzed in the next section.

\section{Gauge field equations}

As pointed out in the previous section it is tempting to consider $A_{\mu}$ and $F_{\mu \nu}$ already as a true gauge potential and a true gauge-field strength. This temptation becomes even higher, since we indeed already know a field which is describable by a tensor of type (7) and a corresponding potential with gauge freedom (3). In this respect there also exists a true field charge, which we denote by $q^{(f)}$. This theory, of course, is MAXWELL's electrodynamics.

As a propagating field, the electromagnetic field $F_{\mu \nu}$ obeys MAXwELL's vacuum equations which come in two pairs. The first pair can be derived from (7) as a mere BIANCHI identity

$$
\partial_{[\mu} F_{\nu \rho]}=0
$$

The second pair equations are given by

$$
\partial_{\mu} F^{\mu \nu}=0
$$

and turn out as the EULER-LAGRANGE equations of

$$
\mathcal{L}_{M}=-\frac{1}{4} F_{\mu \nu} F^{\mu \nu}
$$

Indeed, the MAXwELL Lagrangian $\mathcal{L}_{M}$ is gauge invariant under (3) and most textbooks insist on the statement that (14) is the simplest Lagrangian with this property. Moreover, under the usual field theoretic requirements such as limitation to second order field equations, for instance, (14) may even turn out uniquely. But still, from the GP alone we are not forced to consider the bundle curvature $F_{\mu \nu}$ as a true electromagnetic field with its own kinematics (13). Rather, to describe the field dynamics we have to introduce field sources. In MAXWELL's theory these are the electric charges. Written in LORENTZ covariant notation the field charges are represented by a four current density

$$
J_{\mu}^{(f)}=\rho^{(f)} v_{\mu}
$$

with $\rho^{(f)}$ denoting the density of the field charge $q^{(f)}$ per volume element $V$ and $v_{\mu}$ as the four velocity of $V$. We get a dynamical coupling of the form

$$
\mathcal{L}_{\text {int }}^{(f)}=-\jmath_{\mu}^{(f)} A^{\mu} .
$$


Thus, the EuleR-LAGRANGE equations for the dynamical Lagrangian of electrodynamics $\mathcal{L}_{E D}=$ $\mathcal{L}_{M}+\mathcal{L}_{\text {int }}^{(f)}$ become

$$
\partial^{\mu} F_{\mu \nu}=j_{\nu}^{(f)}
$$

To be sure, Lagrangians (10) and (16), which describe the coupling, have indeed the same structure, and it is very tempting to make the identification

$$
\mathcal{L}_{\text {int }}^{(i)}=\mathcal{L}_{\text {int }}^{(f)} \equiv \mathcal{L}_{\text {int }} .
$$

This would allow us to combine the vacuum field Lagrangian $\mathcal{L}_{M}$ with the coupled matter-field Lagrangian $\mathcal{L}_{D}^{\prime}$ to get the total Lagrangian of the DIRAC-MAXWELL theory

$$
\mathcal{L}_{D M}=\mathcal{L}_{D}+\mathcal{L}_{\text {int }}+\mathcal{L}_{M}
$$

In fact, $\mathcal{L}_{D M}$ describes new physics compared to $\mathcal{L}_{D}$ or $\mathcal{L}_{D}^{\prime}$, respectively. The entire DIRACMAXWELL theory represents the properly combined dynamics of matter-field and gauge-field the key feature of gauge-field theories. Nonetheless, nothing in the argument structure of the GP forces us to make the crucial identification (18) based on the assumption of the equivalence

of $\jmath_{\nu}^{(i)}$ and $\jmath_{\nu}^{(f)}$. Admittedly, the GP works nicely as a heuristic guiding principle to introduce the field Lagrangian and its dynamical coupling. But we are not allowed to sweep the quite different origin of currents (8) and (15) under the rug, for this is far too strong a physical assumption, which does not flow out of a principle of merely conventional character - such as the GP.

Thus, in order to obtain the full DiRAC-MAXwell theory (19) we need a truly physical principle. Otherwise, there remains a "missing link" - at least from the foundational point of view. In search for such a linking principle it might be useful to take a lesson from general relativity, first.

\section{The lesson from general relativity}

\subsection{The gauge theoretic analogy}

General relativity theory (GR) is the commonly accepted theory of gravitation. As it stands it does not have a completely consistent gauge-field theory structure, but rather shows the most important gauge theoretic features such as a principal fiber bundle geometry ${ }^{0}$ and - crucial for our considerations - an underlying gravitational GP. The most distinctive feature of gravitation compared to other gauge field theories is, besides its classical rather than quantum nature, the so-called soldering of the bundle base space and its fibers: in gravitational theories the bundle geometry is inseparably connected to the geometry of the base space. In other words, gravitation is the field which represents the metric properties of spacetime itself (rather than of some internal symmetry space). For our purposes, however, it is sufficient to recall the gauge theoretic analogy of GR.I

\footnotetext{
${ }^{6}$ In standard GR the principal bundle is the bundle of orthonormal frames (tetrad bundle), the associated vector bundle is the tangent bundle of the pseudo-RIEMANNian spacetime manifold (TRAUTMAN 1980).

${ }^{7}$ For a philosophical account on gravitational gauge theories see EYNCK and LYRE (2001); the "ultimate" physical reference is HEHL et al. (1995).
} 
Let us try to mimic a suitable gravitational GP. We start with flat Minkowski space $\mathbb{R}^{(1,3)}$, i.e. the interaction-free case in which no gravitation exists. Hence, we consider the free geodesic equation

$$
m^{(i)} \frac{d v^{\mu}(\tau)}{d \tau}=0
$$

The four vector $v^{\mu}(\tau)=\frac{d x^{\mu}(\tau)}{d \tau}$, tangent to a timelike curve $x^{\mu}(\tau)$, denotes the velocity of a (pointlike) particle with inertial mass $m^{(i)}$. Note that $v^{\mu} \equiv \theta_{0}^{\mu}$ together with a system of three spacelike vectors $\theta_{i}^{\mu}$ forms a tetrad $\theta_{\alpha}^{\mu}=\left(\theta_{0}^{\mu}, \theta_{1}^{\mu}, \theta_{2}^{\mu}, \theta_{3}^{\mu}\right)$. A tetradial reference frame may be considered as representing an observer in spacetime. Clearly, (20) is globally invariant under the homogeneous LORENTZ group $S O(1,3)$ (in principle, other groups are possible as well, such as the group of Poincaré translations $\mathbb{R}^{(1,3)}$, the diffeomorphism group $\mathcal{D}$ iff $(\mathcal{M})$ etc.). Now, the local gauge postulate amounts to

$$
\theta_{\alpha}^{\mu}(\tau)=\hat{L}_{\alpha}^{\beta}(x) \theta_{\beta}^{\mu}(\tau)=e^{\left(\hat{M}^{a}\right)_{\alpha}^{\beta} \Lambda^{a}(x)} \theta_{\beta}^{\mu}(\tau)
$$

with the 6 generators $\hat{M}^{a}$ of $S O(1,3)$ and corresponding local, i.e. spacetime-dependent, transformation parameters $\Lambda^{a}(x)$ and $x \equiv x^{\mu}(\tau)$. In the geodesic equation of (21) we substitute

$$
\left(\hat{M}^{a}\right)_{\alpha}^{\beta} \frac{d}{d \tau} \Lambda^{a}(x)=\left(\hat{M}^{a}\right)_{\alpha}^{\beta} \partial_{\nu} \Lambda^{a}(x) \frac{d}{d \tau} x^{\nu}(\tau)=\Gamma_{\nu \alpha}^{\beta}(x) \frac{d x^{\nu}(\tau)}{d \tau} .
$$

$\Gamma_{\nu}$ must itself satisfy local $S O(1,3)$ gauge transformations

$$
\Gamma_{\nu \alpha}^{\gamma}(x)=\hat{L}_{\alpha}^{\gamma}(x) \Gamma_{\nu \gamma}^{\delta}(x)\left(\hat{L}^{-1}\right)_{\delta}^{\gamma}(x)-\hat{L}_{\alpha}^{\delta}(x) \partial_{\nu}\left(\hat{L}^{-1}\right)_{\delta}^{\gamma}(x)
$$

We insert (21) and (23) in (20) to get the geodesic equation in curved space-time

$$
m^{(i)} \frac{d}{d \tau} \theta_{\alpha}^{\mu}(\tau)+m^{(i)} \Gamma_{\nu \alpha}^{\beta} \frac{d x^{\nu}(\tau)}{d \tau} \theta_{\beta}^{\mu}(\tau)=0 .
$$

In other words, the gauge postulate concerning local LORENTZ rotations of the tetrads leads to a covariant derivative $\nabla_{\tau}$, such that

$$
\frac{d}{d \tau} \theta_{\alpha}^{\mu}(\tau) \rightarrow \nabla_{\tau} \theta_{\alpha}^{\mu}(\tau)=\frac{d}{d \tau} \theta_{\alpha}^{\mu}(\tau)+\Gamma_{\nu \alpha}^{\beta} \frac{d x^{\nu}(\tau)}{d \tau} \theta_{\beta}^{\mu}(\tau)
$$

It is now straightforward to compare GR with the DIRAC-MAXWELL gauge theory as discussed above. Obviously, in the gravitational case the gauge potentials are given by the LEVICivita connection $\Gamma_{\mu}$ (with CHRISTOFfel symbols as its components). From the gravitational potential we may form the curvature tensor $R_{\mu \nu \beta}^{\alpha}$, the so-called RIEMANN tensor, which represents the gravitational field strength. Is it, then, justified to interpret (24) as the geodesic equation in curved spacetime - and, thus, to interpret $\Gamma_{\mu}$ as a true gravitational potential which gives rise to a true gravitational field strength? Of course, the answer can be no other than

\footnotetext{
${ }^{8}$ We use three types of indices:

1. curved (holonomic) LorEnTz indices $\mu, \nu \ldots$ in the space-time manifold (external space),

2. flat (anholonomic) tetrad indices $\alpha, \beta \ldots$ in local Minkowski space (internal space), and

3. the Latin index $a$ in the LIE algebra of the gauge group.
} 
negative! Actually, in GR it is even easier to realize the purely conventional character of the connection occuring in the GP: it is already very well-known that non-vanishing CHRISTOFFELsymbols may occur simply because of some peculiar choice of coordinates (i.e. curvilinear coordinates in flat space). Of course, this does not mean that we already have non-vanishing curvature and, thus, a true gravitational field. Things are more obvious in GR than in gauge theories in general since the intrinsic gauge theoretic conventionalism applies to external rather than to internal coordinate transformations (again, a peculiarity of the bundle soldering). Thus, from the mere logic of the GP we still remain in a type 1 case: flat connections with vanishing fields. It is therefore necessarry to obtain new physics by appealing to a real physical principle which GR is based upon: the principle of equivalence of inertia and gravitation.

\subsection{The equivalence principle}

In NEWTONian physics mass appears in three different meanings, firstly, as a source of the gravitational potential, the so-called active mass $m^{(a)}$ in

$$
\Phi_{g}=-\frac{m^{(a)}}{r}
$$

secondly, as a quantity on which gravitation acts, the so-called passive $\operatorname{mass} m^{(p)}$ in

$$
\vec{F}=-m^{(p)} \vec{\nabla} \Phi_{g}
$$

and, thirdly, as the tendency of resistance of motion, the so-called inertial mass $m^{(i)}$ in

$$
\vec{F}=m^{(i)} \ddot{\vec{r}}
$$

Due to "actio=reactio" the equality of active and passive mass, identified as the so-called gravitational mass $m^{(g)}$, is concluded

$$
m^{(a)}=m^{(p)} \equiv m^{(g)} .
$$

The equality of gravitational and inertial mass, however, does not follow from the NEWTONian axioms. In other words, the empirical fact of the mass-independence of the free fall of ponderable matter remains unexplained

$$
\ddot{\vec{r}}=-\underbrace{\frac{m^{(g)}}{m^{(i)}}}_{=1} \vec{\nabla} \Phi_{g} .
$$

As is well-known, EINSTEIN took this empirical fact very serious: an observer in a freely falling elevator does not feel his own weight. Also, feeling weight is not sufficient to tell him whether this is due to an acceleration of the elevator or due to the existence of an external (homogeneous) gravitational field. Thus, inertia and gravitation, EINSTEIN concluded, are identical in essence (in German: "wesensgleich"). The equivalence principle (EP) acquires the following form: There always exists a local frame of reference, such that the gravitational field vanishes.

I should note two points: firstly, the above formulation is stronger than the empirical universality of the free fall. The latter merely requires that in a gravitational field all (point) particles follow the same path irrespective of their mass. This statement is sometimes called the weak EP. 
As opposed to this, EINSTEIN's EP is a strong EP. It includes the weak version, but, moreover, states that gravity has no influence on any physical process whatsoever. In the following, we will use this version (simply refering to it as EP). Now, secondly, the above formulation contains special relativity as a limiting case. As we will see shortly, this becomes a cruicial feature for generalizing the EP to gauge theories.

But another step first: We are now prepared to return to our question at the end of the preceeding subsection. It is indeed the EP which allows for an interpretation of the LEVICIVITA connection $\Gamma_{\mu}$ as a true gravitational potential in the general case. By analogy with section 3 we must consider the gravitational field with its own field dynamics given by the EINSTEIN field equations

$$
R_{\mu \nu}-\frac{1}{2} R g_{\mu \nu}=-\kappa T_{\mu \nu}
$$

Analogous to the inhomogeneous MAxwelL equations (17), the l.h.s. of (31) is made of curvature (and its contractions, respectively).9 The r.h.s. of both equations represents the field source. Thus, the gravitational mass $m^{(g)}$, which is encoded in the energy-momentum tensor $T_{\mu \nu}$, acts as the field charge of gravity. Due to the equivalence of inertial and gravitational mass,

$$
m^{(i)}=m^{(g)},
$$

we are now able to combine the geodesic equation of motion (24), which includes $m^{(i)}$, with the EInstein field equations (31), which include $m^{(g)}$. From the conceptual point of view, the identification (32) - a direct result of the EP - is the one and decisive link between the equation of motion, which originates from the GP, and the field equations, which are merely motivated by the GP in their formal structure. Due to the EP the LEVI-CIVITA connection may be identified for the general case with a non-flat connection representing a true gravitational potential with non-vanishing fields, i.e. curvature. In other words, only the EP allows for type 2 bundles to enter gravitational theories.

\section{A generalized equivalence principle}

\subsection{The "missing link"}

From all of the above it seems very natural to ask whether there exists a principle in gauge theories in general, which, like the EP in gravitational theories, links up equations of motion and field equations. And if so, maybe it can even be formulated in such a way that the gravitational EP turns out as a special case for gravitational gauge theories. It is the final purpose of this paper to demonstrate exactly this statement: the idea of a generalized equivalence principle.

\footnotetext{
${ }^{9}$ To be sure, the analogy is incomplete. The GR Lagrangian $\mathcal{L}_{G R}=\frac{1}{2 \kappa} \sqrt{-g} R+\mathcal{L}_{\text {Matter }}$ is linear and not quadratic in the fields, compared to (14). It is possible, however, to have a full analogy to the general gauge theoretic YANG-MILLS approach (cf. section 5.2) in the framework of an alternative gravitational gauge theory of the full PoINCARÉ group with a quadratic field Lagrangian including curvature and torsion (HEHL et al. 1980). Standard GR, however, does neither consistently fit into this picture, nor does the equation of motion (24) arise from a genuine field theoretic Lagrangian density with an action functional $\mathcal{S}=-m^{(i)} \int d x^{4} \mathcal{L}$ (such as 1 ), for instance), but rather $\mathcal{S}=-m^{(i)} \int d \tau \sqrt{g_{\mu \mu} v^{\mu} v^{\nu}}$; see also EYNCK and LYRE (2001).
} 
Recall the prerelativistic analogy of NEwTONian gravitation and electrodynamics. If we simply replace the gravitational potential $\Phi_{g}$ by the Coulomb potential $\Phi_{e}$, we may introduce an active electric charge, $\Phi_{e}=-\frac{q^{(a)}}{r}$, analogous to (26) as well as a passive one, $\vec{F}=-q^{(p)} \vec{\nabla} \Phi_{e}$, analogous to (27) and, again due to actio=reactio, we identify

$$
q^{(a)}=q^{(p)} \equiv q^{(f)},
$$

where $q^{(f)}$ is the field charge occuring in section 20. Quite generally, $q^{(f)}$ denotes the field source of the gauge-fields and, hence, gravitational mass may be looked upon as the special case for gravitational interaction.

We must now ask for a generalization of $m^{(i)}$. Is it conceivable to have something like an "inertial charge"? In quantum gauge-field theories the equations of motion originate from the DIRAC Lagrangian (11). This is the reason why the $q^{(i)}$-notation was chosen already in equations (21), (5) and (8). Curiously, the inertial charge implicitly occurs as a factor in the phase of the matter wavefunction $\psi e^{i q^{(i)} \Lambda}$. Due to the intrinsic gauge theoretic conventionalism this factor remains purely conventional without any physical significance up to the point when we plug in the crucial identification

$$
q^{(i)}=q^{(f)}
$$

This statement, in analogy to (32), originates from what I propose to call a generalized EP. Here is a formulation equivalent to the strong version of the gravitational EP:

Generalized equivalence principle (GEP): It is always possible to perform a local gauge transfomation such that, locally (i.e. at a point), the gauge-field vanishes.

Thus, any gauge-field theory includes the interaction-free theory as a local limiting case. The above formulation of the GEP implies that the local gauge transformations are now considered as irrevocably connected with the occurrence of an interaction field, which has its origin in the field charges and obeys its own dynamics. In other words: As far as solely the GP is concerned, local gauge transformations must be considered as bundle coordinate transfromations. Hence, the GP merely amounts to a passive interpretation of local gauge transfomations. As soon as we take the GEP, a truly physical principle, under consideration, too, we are forced to interpret local gauge transfomations actively:ए

\subsection{Yang-Mills theories, charges and couplings}

So far, I have solely discussed the DIRAC-MAXwELL theory (or QED, respectively) as well as the GR analogue as gauge theories. However, the quantum gauge theories of the standard model are non-abelian gauge theories, known as YANG-MiLls theories. I shall show now that the above considerations apply to YANG-MILLS theories in general. The non-abelian groups occuring in

\footnotetext{
${ }^{10}$ In more detail, Michael Redhead (1998) has discussed certain problems arising in connection with active and passive interpretations of local gauge transformations. It seems very likely that our presentation sheds new light on his discussion in favour of an active interpretation of gauge transformations. This will be the task of a special paper.
} 
the standard model are $S U(2)_{F}$ for flavour charge (weak isospin) and $S U(3)_{C}$ for color. In the general $S U(n)$-case, the gauge postulate leads to local gauge transformations

$$
\Psi(x) \rightarrow \Psi^{\prime}(x)=e^{i g^{(i)} \Lambda^{a}(x) \hat{t}^{a}} \Psi(x) \equiv \hat{U}(x) \Psi(x)
$$

instead of (2), where $\Psi=\left(\ldots, \psi^{i}, \ldots\right)^{T}, i=1,2 \ldots n$ denotes the fundamental spinor representation of $S U(n)$ with $a=1,2 \ldots n^{2}-1$ generators $\hat{t}^{a}$. As a generalization of (四) we get

$$
B_{\mu}^{\prime a}(x) \hat{t}^{a}=\hat{U}(x) B_{\mu}^{a}(x) \hat{t}^{a} \hat{U}^{+}(x)-\frac{i}{g^{(i)}} \hat{U}(x) \partial_{\mu} \hat{U}^{+}(x) .
$$

The covariant derivative reads

$$
D_{\mu}=\partial_{\mu}+i g^{(i)} B_{\mu}^{a} \hat{t}^{a} .
$$

We may, again, construct a field strength tensor analogous to (7)

$$
F_{\mu \nu}^{a} \hat{t}^{a}=-\frac{i}{g^{(i)}}\left[D_{\mu}, D_{\nu}\right]=\left(\partial_{\mu} B_{\nu}^{a}-\partial_{\nu} B_{\mu}^{a}-g^{(i)} f^{a b c} B_{\mu}^{b} B_{\nu}^{c}\right) \hat{t}^{a} .
$$

From global gauge covariance under $S U(n)$ we get $n^{2}-1$ conserved NoETHER currents

$$
\jmath_{\mu}^{(i) a}=g^{(i)} \bar{\Psi} \gamma_{\mu} \hat{t}^{a} \Psi
$$

with a continuity equation

$$
\left(D^{\mu} \jmath_{\mu}^{(i)}\right)^{a}=\partial^{\mu} \jmath_{\mu}^{(i) a}-g^{(i)} f^{a b c} B^{\nu b} j_{\nu}^{(i) c}=0 .
$$

Comparing this equation with its abelian counterpart (9) we can read off the decisive difference between abelian and non-abelian gauge theories: in the latter not only the matter-field current is conserved, there rather exists a contribution from the gauge potentials $B_{\mu}^{a}$ which carry charge. Also, the third term in (38) represents the self-interaction of the YANG-MILLS gauge-bosons.

In analogy to the QED case we have introduced an inertial charge $g^{(i)}$ (or rather a coupling constant, see remarks below). But we still do not have any field equations, since the curvature tensor (38) is not necessarly non-zero from the GP's point of view. Instead, we must introduce the so-called YANG-MiLls equations, generalizations of the MAXwelL equations (17),

$$
\left(D^{\mu} F_{\mu \nu}\right)^{a}=\partial^{\mu} F_{\mu \nu}^{a}-g^{(f)} f^{a b c} B^{\mu b} F_{\mu \nu}^{c}=\jmath_{\nu}^{(f) a} .
$$

There is also a BIANCHI identity analogous to (12), and a YANG-MiLLS Lagrangian $\mathcal{L}_{Y M}$ analogous to (14). In (17), there arises a field charge $g^{(f)}$. To be sure, from the point of view of pure YANG-MiLLS field theory formulae (36) and (38), which also arise there, should then be written with $g^{(f)}$, either. The identification

$$
g^{(i)}=g^{(f)}
$$

expresses the GEP. Only on this basis are we allowed to combine both theories entering a true DiRAC-YANG-MiLls gauge theory $\mathcal{L}_{D Y M}=\mathcal{L}_{D}^{\prime}+\mathcal{L}_{Y M}$.

It may be useful to recall the different meanings of charges and coupling constants in field physics. In QED, for instance, the electric charge $q$ is measured in units $e$, the elementary 
charge W At the same time, $e$ plays the role of the electromagnetic coupling constant. In YANGMills theories, however, we discover a so-called universality of the coupling. This includes two points: firstly, the coupling of the $B_{\mu}^{a}$ gauge potential to the matter-field current (39) has the same strength as the $B_{\mu}^{a}-B_{\mu}^{a}$ coupling, i.e. the coupling of the charged gauge bosons among each other. Secondly, any particle, which couples weakly or strongly, couples with the same interaction strength. This means that there are no multiples of weak or color charges. Interestingly, the first point appears now as a consequence of the GEP rather than the GP (although, the GP would be impossible otherwise). The second point does not follow from any of both principles. For in QED there are "multiples", more precisely thirds, of $e$ : the electromagnetic coupling is $-\frac{1}{3} e$ for d-s-b-quarks, $\frac{2}{3} e$ for u-c-t-quarks, and $1 e$ for electrons, muons or taus.

An explanation of this latter point may rather be due to a proper understanding of the quantum nature of the fundamental charges. With respect to this, consider gravitational theories. It seems that we may clearly distinguish between mass as the charge of gravity and the coupling constant $\kappa=8 \pi G$. But a simple reason for this could be the fact that we are still dealing with a classical theory, i.e. we have not quantized mass. If so, we perhaps would be able to introduce an analogue of $e$ (or $\frac{1}{3} e$, respectively) such as the PLANCK mass, for instance. But these questions are certainly beyond the scope of this paper.

\subsection{Experimental conclusions}

By its very nature the equivalence principle must be checked empirically, since it is the one and decisive empirical input to gauge theories. Indeed, the identification (34) turns out as a consequence of the GEP. The reason is that if we regard the connection field as truly physical, it must have its sources in the field charges. But this exactly means that we have to identify the $q^{(i)}$ factor arising in the transformation (2) with the field charge $q^{(f)}$. Now, if we assume, for a moment, the ratio of $q^{(f)}$ and $q^{(i)}$ to deviate from 1, then this means that different types of particles of equal electric charge would couple differently to the electromagnetic field. Hence, we should expect a difference in the coupling of electrons and muons, d-quarks and s-quarks etc. But this is certainly not what we observe! Therefore, the GEP predicts a whole variety of null-experiments.

Note that the analogy to the gravitational case is quite straightforward. We must check empirically that different materials do have the same free fall behaviour. Otherwise, the $m^{(g)}$ $m^{(i)}$-ratio in (30) would lead to different results for, say, 1 kilogram of wood than for 1 kilogram of iron. In the same sense as this possibility, from a priori grounds, cannot be excluded, the coupling of different types of particles with the same gauge field charge, on principle, cannot be excluded. In other words, the GEP is equivalent to the statement that all types of particles with the same $q^{(f)}$ show the same coupling. Otherwise, we would have to write down a different DIRAC equation

$$
\left(i \gamma^{\mu} \partial_{\mu}-m\right) \psi e^{i q^{(i)} \Lambda}=c_{p} q^{(f)} \gamma^{\mu} A_{\mu} \psi e^{i q^{(i)} \Lambda}
$$

\footnotetext{
${ }^{11}$ More advanced, one uses dimensionless coupling strengths, such as $\alpha \sim \frac{1}{137}$ for QED or $\alpha_{s} \sim \frac{1}{10}$ for QCD. Of course, in physics only the dimensionless ratios of the fundamental parameters such as masses and charges will have any convention-independent significance.
} 
for different types of particles with the same $q^{(f)}$, but with a particle type-dependent factor $c_{p}$. [2] High energy physics tells us that this is, of course, not the case; and therefore we may safely say that the GEP is empirically very well confirmed.

\section{Concluding remarks}

It was the purpose of this paper to convince the reader that the celebrated GP cannot take the burden of a full derivation of gauge field theories. Rather, the crucial gauge-theoretic combination of a pure matter-field and a pure gauge-field theory, i.e. $\mathcal{L}_{\text {total }}=\mathcal{L}_{D}^{\prime}+\mathcal{L}_{G F}$, should be based on a second principle, a generalized equivalence principle. This is a new idea, which of course does not change the practical application of gauge theories, but rather affects their deeper conceptual understanding.

The idea of postulating an EP as a general principle in gauge theories has already been expressed by Gerhard MACK (1981). However, as far as I can see, his arguments have a quite different origin: MACK seems to be motivated by the close analogy to GR. Certainly, he did not stress the intrinsic gauge theoretic conventionalism. I do not see, however, that there is anything in his presentation which must convince the reader to apply his EP beyond the GP, if one does not make the conventionalism claim.

From the standpoint of philosophy of physics, Harvey Brown (1999) and Paul Teller (2000a) have emphasized that the gauge argument applies to a mere coordinate transformation. Thus, in a way they also take the gauge theoretic conventionalism serious. Moreover, TELLER (2000b) pointed out that from the mere GP the QED connection appears to be only the $A_{\mu}$ vector field rather than $q A_{\mu}$. He then concludes that the GP does not lead to a derivation of the charge $q$ (or coupling constant $e$, respectively). I do not follow the whole variety of suggestions which he draws from his analysis, but certainly he comes very close to my point. From this paper's perspective, of course, one could argue the following way: there is a factor, say $q^{(i)}$, which is not determined from the gauge argument. On the other hand, there is a MAXwELL field source $q^{(f)}$. But we will know about their identification from experiment only.

Hence, the GEP stresses the one and decisive empirical input for considering gauge theories as physical theories.

\footnotetext{
${ }^{12}$ Of course, the situation is more confusing in the quantum gauge theoretic case due to the occurence of $q^{(i)}$ in the phase of the matter-wavefunction $\psi e^{i q^{(i)} \Lambda}$. Conceptually I decidedly agree with what SuNNY AUYANG (1995) has pointed out: in modern field theories the very notion of an event becomes inseparably connected with the idea of an interaction. The inertial charge seems to be no direct observable (in contrast to inertial mass). However, for the formulation of the GEP it is sufficient to consider the ratio of $q^{(i)}$ and $q^{(f)}$.
} 


\section{Acknowledgements}

Special thanks to M. Drieschner, T. O. Eynck, D. Graudenz, M. Kuhlmann and M. TIELKE for helpful discussions and several valuable comments on earlier versions of the manuscript.

\section{References}

Auyang, S. Y. (1995). How is Quantum Field Theory Possible? Oxford University Press, New York.

Brown, H. R. (1999). Aspects of Objectivity in Quantum Mechanics. In ButTerfield, J. and C. PAgonis, editors. From Physics to Philosophy. Cambridge University Press, Cambridge.

Eynck, T. O. and LyRe, H. (2001). How to Gauge Gravity? Underdetermination in Gravitational Theories. In preparation.

Guttmann, Y. M. and Lyre, H. (2000). Fiber Bundle Gauge Theories and "Field's Dilemma". (E-print arXiv:physics/0005051).

Hehl, F. W., McCrea, J. D., Mielke, E. W., and Ne'eman, Y. (1995). Metric-affine gauge theory of gravity: Field equations, Noether identities, world spinors, and breaking of dilation invariance. Physics Reports, 258:1-171.

Hehl, F. W., Nitsch, J., and Heyde, P. V. D. (1980). Gravitation and the Poincaré gauge field theory with quadratic Lagrangian. In HELD (1980).

Held, A., editor (1980). General Relativity and Gravitation: One Hundred Years after the Birth of Albert Einstein. Plenum Press, New York. 2 Volumes.

Lyre, H. (1999). Gauges, Holes, and their 'Connections'. In Howard, D., editor. Proceedings of the "Fifth International Conference on the History and Foundations of General Relativity", Notre Dame, Indiana. (E-print arXiv:gr-qc/9904036).

Mack, G. (1981). Physical principles, geometrical aspects, and locality properties of gauge field theories. Fortschritte der Physik, 29:135-185.

Redhead, M. (1998). Review: S. Y. Auyang, How is Quantum Field Theory Possible? (Oxford, Oxford University Press, 1995). The British Journal for the Philosophy Science, 49:499-507.

Teller, P. (2000a). The gauge argument. Philosophy of Science, 67(3, Supplement):S466-S481. (PSA98 Proceedings, part II, ed. by D. A. HowARD).

Teller, P. (2000b). Some questions about the QED coupling constant. Preprint.

Trautman, A. (1980). Fiber bundles, gauge fields, and gravitation. In Held (1980). 